\title{
Effect of alteplase on the CT hyperdense artery sign and outcome after ischemic stroke
} OPEN

Grant Mair, MBChB

Rüdiger von Kummer,

ProfDrmed

Zoe Morris, MBBS

Anders von Heijne, MD

Nick Bradey, MBBS

Lesley Cala, MD

André Peeters, MD

Andrew J. Farrall, MD

Alessandro Adami, MD

Gillian Potter, MD

Geoff Cohen, MSc

Peter A.G. Sandercock,

DM

Richard I. Lindley, MD

Joanna M. Wardlaw, MD

For the IST-3

Collaborative Group

Correspondence to

Prof. Wardlaw:

joanna.wardlaw@ed.ac.uk

Supplemental data at Neurology.org

\section{ABSTRACT}

Objective: To investigate whether the location and extent of the CT hyperdense artery sign (HAS) at presentation affects response to IV alteplase in the randomized controlled Third International Stroke Trial (IST-3).

Methods: All prerandomization and follow-up (24-48 hours) CT brain scans in IST-3 were assessed for HAS presence, location, and extent by masked raters. We assessed whether HAS grew, persisted, shrank, or disappeared at follow-up, the association with 6-month functional outcome, and effect of alteplase. IST-3 is registered (ISRCTN25765518).

Results: HAS presence (vs absence) independently predicted poor 6-month outcome (increased Oxford Handicap Scale [OHS]) on adjusted ordinal regression analysis (odds ratio [OR] 0.66, $p<0.001$ ). Outcome was worse in patients with more (vs less) extensive HAS (OR 0.61, $p=$ $0.027)$ but not in proximal (vs distal) HAS $(p=0.420)$. Increasing age was associated with more HAS growth at follow-up (OR 1.01, $p=0.013)$. Treatment with alteplase increased HAS shrinkage/disappearance at follow-up (OR 0.77, $p=0.006$ ). There was no significant difference in HAS shrinkage with alteplase in proximal (vs distal) or more (vs less) extensive HAS ( $p=0.516$ and $p=$ 0.580 , respectively). There was no interaction between presence vs absence of HAS and benefit of alteplase on 6-month OHS ( $p=0.167)$.

Conclusions: IV alteplase promotes measurable reduction in HAS regardless of HAS location or extent. Alteplase increased independence at 6 months in patients with and without HAS.

Classification of evidence: This study provides Class I evidence that for patients within 6 hours of ischemic stroke with a CT hyperdense artery sign, IV alteplase reduced intra-arterial hyperdense thrombus. Neurology ${ }^{\circledR}$ 2016;86:118-125

\section{GLOSSARY}

ACA = anterior cerebral artery; $\mathbf{C l}=$ confidence interval; HAS = hyperdense artery sign; $\mathbf{H U}=$ Hounsfield units; ICA = internal carotid artery; IST-3 = Third International Stroke Trial; $\mathbf{M C A}=$ middle cerebral artery; NIHSS = NIH Stroke Scale; OHS = Oxford Handicap Scale; $\mathbf{O R}=$ odds ratio; PCA = posterior cerebral artery; SIRS = Systematic Image Review System.

Arterial hyperattenuation on noncontrast CT, the hyperdense artery sign (HAS), is a consistently recognized CT sign of acute ischemic stroke. ${ }^{1}$ HAS is highly specific and moderately sensitive for intracranial arterial obstruction by thrombus. ${ }^{2}$ HAS is associated with increased stroke severity at presentation and worse long-term outcomes. ${ }^{3-5}$ There are, however, limited data on how the location, extent, or persistence of HAS relates to functional outcome following stroke, and importantly whether patients with (vs without) HAS benefit differently from IV thrombolysis with alteplase. ${ }^{6-9}$

\footnotetext{
From the Division of Neuroimaging Sciences (G.M., Z.M., A.J.F., G.C., J.M.W.) and the Division of Clinical Neurosciences (P.A.G.S.), University of Edinburgh, UK; the Department of Neuroradiology (R.v.K.), Dresden University Stroke Centre, Germany; Danderyd Hospital (A.v.H.), Stockholm, Sweden; Neuroradiology (N.B.), James Cook University Hospital, Middlesborough, UK; School of Pathology and Laboratory Medicine (L.C.), University of Western Australia, Perth; Cliniques Universitaires St Luc (A.P.), Neurologie, Belgium; Stroke Center (A.A.), Sacro Cuore-Don Calabria Hospital, Negrar, Italy; the Department of Neuroradiology (G.P.), Salford Royal NHS Foundation Trust, Manchester, UK; and the Westmead Hospital Clinical School and The George Institute for Global Health (R.I.L.), University of Sydney, Australia.

Coinvestigators are listed on the Neurology ${ }^{\circledR}$ Web site at Neurology.org.

Go to Neurology.org for full disclosures. Funding information and disclosures deemed relevant by the authors, if any, are provided at the end of the article. The Article Processing Charge was paid by the MRC.

This is an open access article distributed under the terms of the Creative Commons Attribution License 4.0 (CC BY), which permits unrestricted use, distribution, and reproduction in any medium, provided the original work is properly cited.
} 
The Third International Stroke Trial (IST-3) was a large $(\mathrm{n}=3,035)$, multicenter, randomized controlled trial testing IV alteplase given within 6 hours of ischemic stroke. ${ }^{10}$ A central masked panel assessed prerandomization and follow-up CT for the presence of HAS.

We analyzed IST-3 imaging data to investigate whether, in this large, prospectively studied group of patients, the presence, location, and extent of HAS was associated with response to IV alteplase assessed as both change in HAS on short-term follow-up and also its effect on 6-month functional outcome.

METHODS IST-3. IST-3 was an international, multicenter, prospective, randomized, open, blinded endpoint (PROBE) trial of IV alteplase in acute ischemic stroke. Enrollment, data collection, image analysis, and CONSORT compliance have been described elsewhere. ${ }^{10,11}$ Briefly, adult patients with acute stroke of any severity, with no upper age limit, were eligible if IV alteplase could be started within 6 hours of stroke onset and CT or MRI had excluded intracranial hemorrhage and any structural stroke mimic. IST-3 used the uncertainty principle for enrollment, i.e., if the randomizing clinician believed that alteplase was clearly indicated or contraindicated, such a patient could not be enrolled; patients were only enrolled when there was genuine uncertainty over the benefit of alteplase for that individual. ${ }^{10,11}$ Stroke severity prior to randomization was assessed with the NIH Stroke Scale (NIHSS). After entry of baseline data, patients were randomized to receive IV alteplase $(0.9 \mathrm{mg} / \mathrm{kg})$ or control. Results were analyzed on an intention-to-treat basis. Patients were followed by postal questionnaire or masked telephone interview at 6 months and functional status assessed with the Oxford Handicap Scale (OHS)

Standard protocol approvals, registrations, and patient consents. Ethical approval for IST-3 was granted by the Scotland A research ethics committee and by local ethics committees. Informed consent was obtained for all patients. IST-3 is registered with Current Controlled Trials, ISRCTN25765518.

Imaging protocol. The IST-3 imaging protocol has been described previously. ${ }^{10,12}$ Briefly, CT scans were required to cover the brain from foramen magnum to vertex, with maximum slice thickness $4-5 \mathrm{~mm}$ through the posterior fossa and $8-10 \mathrm{~mm}$ for the cerebral hemispheres, with no slice gap. Thinner slices were also accepted. Scans were windowed on 80 Hounsfield units (HU) width and center level of 35-40 HU. Follow-up brain imaging (CT or MRI) was also required, to the same protocol, for all patients, between 24 and 48 hours after stroke onset. All imaging was reviewed centrally for quality control and validation.

Image analysis. A centralized panel of neuroradiologists and neurologists experienced in reviewing stroke imaging analyzed all imaging with an online assessment tool, the Systematic Image Review System (SIRS), recording assessments on a validated structured pro forma ${ }^{1,13}$ available at www.sbirc.ed.ac.uk/research/ imageanalysis.html, accessed November 23, 2015. Assessors were masked to all other imaging and clinical data. HAS presence was determined visually (i.e., the reader decided if a vessel appeared hyperdense; no objective HU measurements were made). Where present, the location and extent of HAS was recorded by selecting the 3 largest vessels affected from the following options: internal carotid artery (ICA), middle cerebral artery (MCA) mainstem, sylvian branches of MCA, anterior cerebral artery (ACA), posterior cerebral artery (PCA), vertebral artery, basilar artery. A subgroup of scans $(n=272)$ underwent a second independent read as part of the IST-3 angiography and perfusion imaging substudy. ${ }^{14}$ Secondary reads were performed by a different panel of assessors (see coinvestigator list on the Neurology ${ }^{\circledR}$ Web site at Neurology.org) using SIRS as described above. Scans that were read twice were used to test interrater reliability of HAS assessment.

Data analysis. These analyses are restricted to patients with a noncontrast CT obtained prerandomization. The location of HAS was classified as proximal (ICA, MCA mainstem, vertebral or basilar arteries) or distal (ACA, PCA, or sylvian branches of the MCA). HAS extent was classified by the number of contiguous named vessels involved ( $0,1,2$, or 3 as per the predefined options). We compared prerandomization and follow-up scans in all patients who had noncontrast CT performed at both times and calculated the change in HAS segment number from a minimum of -3 to a maximum of +3 (negative numbers indicate shrinkage and positive numbers indicate growth of HAS).

Statistics. We used univariate and multivariate tests to examine differences between all IST-3 patients and the subset who were HASpositive prerandomization, between the treatment and control groups with HAS, and for associations among the presence, extent, location, and persistence of HAS, baseline clinical features, and effect of alteplase.

For univariate analysis of parametric data, we used $t$ tests to compare ratios and means; for nonparametric data, we used MannWhitney $U$ tests. We calculated $\chi^{2}$ statistics for dichotomous data. We used Krippendorff $\alpha(\mathrm{K}-\alpha)$ to test interrater reliability. K- $\alpha$ results range from -1.0 to +1.0 where +1.0 equates to perfect agreement, 0.0 means no agreement, and -1.0 implies perfect disagreement. ${ }^{15}$

We used multivariate ordinal regression to calculate common odds ratios (ORs) with 6-month OHS and change in HAS segment number as dependent variables. ${ }^{16}$ We tested the effect of HAS presence, extent, and location on outcome in separate models. Consistent with the main IST-3 analyses, ${ }^{10,17}$ multivariate models were adjusted for the effect of patient age, NIHSS, and time from stroke onset to prerandomization scan since these variables predicted outcome in the main trial. ${ }^{10}$ To reduce confounding between HAS extent and location on outcome, the effect of location was assessed only among those with HAS in a single arterial segment. To stabilize ordinal regression estimates, we used the same approach as the original IST-3 analysis of functional outcome, where the more severe grades of OHS (4-6) were grouped as one, leaving 5 ordinal analysis groups. ${ }^{17}$ Similarly, the variable time from stroke onset to prerandomization scan was grouped into 61 -hour windows (0-6 hours), the variable time from prerandomization scan to follow-up scan was grouped into 5 12-hour windows ( $\leq 12,13-24,25-36$, $37-48$, and $>48$ hours) and the variable change in HAS segment number was grouped into 3 outcomes (fewer segments $=$ shrinkage, no change, more segments $=$ growth).

We performed tests of interaction (using Comprehensive MetaAnalysis software; Biostat, Englewood, NJ) to compare ORs for the effect of IV alteplase on change in HAS extent in distal vs proximal HAS and in 1 segment vs $>1$ segment HAS and to compare the effect of alteplase on outcome in those with vs those without HAS.

All other analyses were performed with IBM SPSS statistics software, version 20.0 (IBM Corporation, Armonk, NY). We considered $p<0.05$ significant.

Primary research question. To assess the response of HAS to IV alteplase and to determine if the presence, location, extent, or persistence of HAS modified the effect of alteplase on outcome. 
Classification of evidence. This study provides Class I evidence that IV alteplase $(0.9 \mathrm{mg} / \mathrm{kg})$ given within 6 hours of ischemic stroke onset accelerates shrinkage of HAS at follow-up 24-48 hours later.

RESULTS Demographics and clinical and outcome data. Most IST-3 patients had noncontrast CT performed both prerandomization (2,961/3,035, $97.6 \%)$ and at follow-up $(2,779 / 3,035,91.6 \%) 24$ 48 hours later. MRI was the imaging method in 56 and 151 patients, respectively. Prerandomization or follow-up CT scans were not available for central review in $18(0.6 \%)$ and 105 cases (3.5\%), respectively. Thus, the total number of patients with centrally reviewed CT both prerandomization and at follow-up was 2,731 (90.0\% of 3,035). HAS data were missing for one follow-up CT (poor quality). There were no significant differences in the demographic and clinical data for the 2,961 patients with centrally reviewed prerandomization CT, nor for the 2,731 patients who also had follow-up CT when both groups were compared with 3,035 patients in the full IST-3 trial (data not shown).

The results of interrater reliability are as follows: for HAS identification, $\mathrm{K}-\alpha=0.40$; for assessment of the largest artery involved, $\mathrm{K}-\alpha=0.46$; and for assessment of HAS extent, $\mathrm{K}-\alpha=0.39$.

Of 2,961 patients with prerandomization CT, $716(24.2 \%)$ demonstrated HAS. On univariate analysis, patients with baseline HAS were younger and had a more severe stroke and worse 6-month outcomes than those without HAS (table 1). Among patients with HAS, those allocated control had an increased median time between prerandomization and follow-up scans compared with patients allocated alteplase. None of the other demographic or clinical measures in patients with HAS were significantly different between alteplase and control groups (table e-1).

For the multivariable ordinal analysis testing prerandomization variables associated with 6-month outcome in the whole group (OHS as dependent variable, $\mathrm{n}=2,961$ ), alteplase treatment allocation independently predicted a better outcome (lower OHS); OR 1.29, 95\% confidence interval (CI) $1.12-1.50, p=0.001$. In the same analysis, the presence vs absence of HAS independently predicted a worse outcome (higher OHS, figure e-1); OR 0.66, 95\% CI $0.55-0.80, p<0.001$. These results are adjusted for age, NIHSS, and time from stroke onset to scan (table 2 and figure 1).

Location, extent, and persistence of HAS. For 2,731 patients with CT performed prerandomization and at follow-up, HAS was identified on $674(24.7 \%$ of 2,731) prerandomization and 520 (19.0\%) follow-up scans. In 870 cases (31.9\%), HAS was evident on at least one scan. On univariate analyses, 6-month outcome was worse if HAS was found only in proximal rather than only in distal vessels, if HAS was more vs less extensive, if HAS showed growth rather than shrinkage between scans, if HAS persisted at follow-up rather than disappeared, and if a new HAS developed between scans (table e-2).

For multivariate analyses testing the effect of HAS characteristics on outcome (OHS as dependent variable) among patients with HAS in the whole group $(\mathrm{n}=$ 716), HAS extent $(1,2$, or 3 segments) independently predicted a worse outcome (higher OHS); OR 0.61, 95\% CI 0.39-0.94, $p=0.027$. In patients with HAS in only 1 arterial segment $(\mathrm{n}=589)$, HAS location (proximal vs distal) was not independently associated with outcome, OR 1.17, 95\% CI 0.80-1.71, $p=$ 0.420 . These analyses are adjusted for age, NIHSS, time

Table 1 Demographic and clinical data of IST-3 patients with and without a HAS on prerandomization CT

\begin{tabular}{|c|c|c|c|c|}
\hline & All patients $(\mathrm{n}=2,961)$ & \multicolumn{3}{|c|}{ Comparison of those with and without HAS } \\
\hline Male sex, $n(\%)$ & $1,427(48.2)$ & $356(49.7)$ & $1,071(47.7)$ & 0.348 \\
\hline Stroke onset to prerandomization scan, min, mean (SD) & $163(73)$ & $159(71)$ & $164(73)$ & 0.082 \\
\hline Baseline NIHSS, median (IQR) & $11(6-18)$ & $16(10-20)$ & $9(6-16)$ & $<0.001$ \\
\hline OHS at 6 months, median (IQR) & $4(2-6)$ & $5(3-6)$ & $3(1-5)$ & $<0.001$ \\
\hline Death by 6 months, $\mathrm{n}(\%)$ & 797 (26.9) & $263(36.7)$ & $534(23.8)$ & $<0.001$ \\
\hline Treated with alteplase, $\mathrm{n}(\%)$ & $1,484(50.1)$ & $368(51.4)$ & $1,116(49.7)$ & 0.432 \\
\hline
\end{tabular}

Abbreviations: HAS = hyperdense artery sign; IQR = interquartile range; IST-3 = Third International Stroke Trial; NIHSS = NIH Stroke Scale; OHS = Oxford Handicap Scale.

Analysis restricted to patients with prerandomization CT imaging only $(n=2,961)$. 


\begin{tabular}{|c|c|c|c|c|}
\hline \multirow[t]{2}{*}{ Multivariable analyses testing } & of HAS pres & and locatio & outcome & \multirow[b]{2}{*}{$p$ Value } \\
\hline & Raw data & Odds ratio & $95 \% \mathrm{Cl}$ & \\
\hline \multicolumn{5}{|l|}{ Presence of HAS $(n=2,961)$} \\
\hline Age, $y$ & $81(72-86)$ & 0.96 & $0.96-0.97$ & $<0.001$ \\
\hline NIHSS & $11(6-18)$ & 0.84 & $0.83-0.85$ & $<0.001$ \\
\hline Stroke onset to prerandomization scan, $\mathrm{h}$ & $3(2-4)$ & 1.07 & $1.01-1.14$ & 0.018 \\
\hline HAS present & $n=716$ & 0.66 & $0.55-0.80$ & $<0.001$ \\
\hline Treated with alteplase & $\mathrm{n}=1484$ & 1.29 & $1.12-1.50$ & 0.001 \\
\hline \multicolumn{5}{|l|}{ Extent of HAS $(n=716)$} \\
\hline Age, y & $80(70-85)$ & 0.95 & $0.94-0.96$ & $<0.001$ \\
\hline NIHSS & $16(10-20)$ & 0.84 & $0.82-0.87$ & $<0.001$ \\
\hline Stroke onset to prerandomization scan, $\mathrm{h}$ & $3(2-4)$ & 1.06 & $0.92-1.22$ & 0.412 \\
\hline HAS extent (1-3 segments) & $1(1-1)$ & 0.61 & $0.39-0.94$ & 0.027 \\
\hline Treated with alteplase & $n=368$ & 1.06 & $0.76-1.48$ & 0.714 \\
\hline \multicolumn{5}{|l|}{ Location of HAS $(n=589)$} \\
\hline Age, $y$ & $81(71-85)$ & 0.95 & $0.94-0.97$ & $<0.001$ \\
\hline NIHSS & $15(10-20)$ & 0.84 & $0.81-0.87$ & $<0.001$ \\
\hline Stroke onset to prerandomization scan, $\mathrm{h}$ & $3(2-4)$ & 1.03 & $0.89-1.20$ & 0.681 \\
\hline HAS in distal location (rather than proximal) & $\mathrm{n}=182$ & 1.17 & $0.80-1.71$ & 0.420 \\
\hline Treated with alteplase & $\mathrm{n}=301$ & 1.15 & $0.81-1.64$ & 0.442 \\
\hline
\end{tabular}

Abbreviations: $\mathrm{Cl}=$ confidence interval; HAS = hyperdense artery sign; NIHSS = NIH Stroke Scale.

Ordinal regression analyses with Oxford Handicap Scale as the dependent variable for each. Presence of HAS = all Third International Stroke Trial patients with prerandomization CT. Extent of HAS = includes only patients with HAS on prerandomization CT. Location of HAS = includes only patients with HAS in a single arterial segment on prerandomization CT. Raw data provided as median (interquartile range) unless otherwise stated. Odds ratio $<1$ indicates worse outcome.

from stroke onset to scan, and treatment allocation (table 2).

Effect of alteplase on HAS. There were univariate associations between alteplase treatment and an increased likelihood of both HAS shrinkage and HAS disappearance $(222 / 440=50.5 \%$ vs $166 / 429=38.7 \%$ shrank, $\chi^{2}=12.6, p=0.002$, and $198 / 350=56.6 \%$ vs $151 /$ $323=46.7 \%$ disappeared, $\chi^{2}=6.5, p=0.011$, respectively). In patients treated with alteplase, distal vs proximal HAS $(65 / 86=75.6 \%$ vs $105 / 198=$ $53.0 \%$, respectively, $\chi^{2}=12.7, p<0.001$ ) and single segment vs multisegment HAS $(170 / 284=59.9 \%$ vs $28 / 66=42.4 \%$, respectively, $\left.\chi^{2}=6.6, p=0.010\right)$ were more likely to have disappeared at follow-up. Fewer patients who received alteplase had developed a new HAS in the interim between pre-randomization and follow-up CT, although the difference was not

Figure 1 Separate ordinal regression analyses show odds ratios for the effect of alteplase treatment on 6 -month functional outcome in the full group $(n=2,961)$ and in the subgroups with and without a hyperdense artery sign (HAS) on prerandomization CT

\begin{tabular}{|c|c|c|c|c|}
\hline Subgroup & $\begin{array}{c}\text { Treated with } \\
\text { alteplase }\end{array}$ & \multicolumn{2}{|c|}{ Odds ratio (95\% confidence interval) } & $\begin{array}{c}\text { Test for } \\
\text { interaction }\end{array}$ \\
\hline \multirow{2}{*}{$\begin{array}{c}\text { HAS } \\
(n=716)\end{array}$} & Yes (368) & \multirow{2}{*}{$1.05(0.75-1.45)$} & & \multirow{6}{*}{$p=0.167$} \\
\hline & No (348) & & & \\
\hline \multirow{2}{*}{$\begin{array}{l}\text { No HAS } \\
(n=2245)\end{array}$} & Yes (116) & \multirow{2}{*}{$1.36(1.16-1.60)$} & & \\
\hline & No (1129) & & & \\
\hline \multirow{2}{*}{$\begin{array}{c}\text { All patients } \\
(n=2961)\end{array}$} & Yes (1484) & \multirow{2}{*}{$1.29(1.12-1.50)$} & & \\
\hline & No (1477) & & & \\
\hline
\end{tabular}

Odds ratio $>1$ (right of line) indicates better outcome (lower 6-month Oxford Handicap Scale). Results for the HAS and no HAS groups are adjusted for the effect of age, time from stroke onset to scan (hours), and NIH Stroke Scale (NIHSS) score. Results for the full group are adjusted for the effect of age, time from stroke onset to scan (hours), NIHSS, and presence/ absence of HAS. 


\begin{tabular}{|c|c|c|c|c|c|}
\hline \multirow[t]{2}{*}{ Table 3} & \multicolumn{5}{|c|}{$\begin{array}{l}\text { Factors associated with change in extent of HAS from } \\
\text { prerandomization to follow-up scan }\left(n=2,730^{\text {a }}\right)\end{array}$} \\
\hline & & Raw data & Odds ratio & $95 \% \mathrm{Cl}$ & $p$ Value \\
\hline Age, y & & 81 (72-86) & 1.01 & $1.00-1.02$ & 0.013 \\
\hline \multicolumn{2}{|c|}{ Baseline NIHSS } & $11(6-18)$ & 1.00 & $0.98-1.01$ & 0.475 \\
\hline \multicolumn{2}{|c|}{$\begin{array}{l}\text { Prerandomization scan to follow-up } \\
\text { scan, } h\end{array}$} & $26(24-36)$ & 0.91 & $0.82-1.00$ & 0.059 \\
\hline \multicolumn{2}{|c|}{ Treated with alteplase } & $n=1397$ & 0.77 & $0.65-0.93$ & 0.006 \\
\hline
\end{tabular}

Abbreviations: $\mathrm{Cl}=$ confidence interval; $\mathrm{HAS}=$ hyperdense artery sign; $\mathrm{NIHSS}=\mathrm{NIH}$ Stroke Scale.

Ordinal regression analysis with change in HAS segment number (shrinkage, no change, growth) as the dependent variable. Raw data provided as median (interquartile range) unless otherwise stated. Time between scans was divided into 5 groups $(\leq 12,13-24,25-36$, $37-48$, and $>48$ hours). Odds ratio $<1$ indicates HAS shrinkage, $>1$ indicates HAS growth. ${ }^{a}$ HAS data were missing for one follow-up CT (poor quality scan).

significant $(90 / 957=9.4 \%$ vs $106 / 904=11.8 \%$, $\left.\chi^{2}=2.2, p=0.143\right)$.

For multivariate analyses, testing factors associated with change in HAS segment number (dependent variable), alteplase treatment was an independent predictor of HAS shrinkage at follow-up (OR 0.77, 95\% CI $0.65-0.93, p=0.006$ ), while HAS growth was more likely in older patients (OR 1.01, 1.00-1.02, $p=0.013$ ) (table 3). Both proximal and distal HAS were equally likely to shrink following alteplase treatment (OR 0.66, 95\% CI 0.45-0.98, and OR 0.51, 95\% CI 0.26-1.00, respectively), with no evidence of an interaction between HAS location and alteplase effect ( $p=0.516$ ) (figure 2). HAS affecting a single arterial segment prerandomization was more likely to shrink following alteplase treatment (OR 0.60, 0.43 0.85 ), but alteplase was not an independent predictor of HAS shrinkage if more than 1 segment was affected prerandomization (OR 0.78, 95\% CI 0.33-1.86). Nevertheless, there was no evidence of an interaction between HAS extent and alteplase effect $(p=0.580)$ (figure 2). These results are adjusted for patient age, time (between prerandomization and follow-up scans), and stroke severity.

Effect of alteplase on patient outcome in those with HAS. Results of multivariate analysis of alteplase effect on outcome (OHS as dependent variable) for the subgroups with (OR 1.05, 0.75-1.45) and without (OR 1.36, 1.16-1.60) HAS on prerandomization CT are shown in figure 1 . There was no evidence of an interaction with the presence vs absence of HAS $(p=0.167)$.

DISCUSSION In this analysis of IST-3, we show that IV alteplase both reduces the persistence (more likely to shrink, or disappear) and limits the formation (less likely to grow) of HAS compared to patients allocated control. We found that alteplase increases HAS shrinkage/disappearance independent of age, baseline stroke severity, and time between prerandomization and follow-up scans. While HAS disappearance following treatment with alteplase was more common for small or distal vs large or proximal HAS on univariate analysis, the alteplase effect on HAS reduction/shrinkage was similar across all subgroups on multivariate regression analysis. We found no difference in the likelihood of HAS shrinkage for proximal vs distal HAS or for single segment vs multisegment HAS, i.e., alteplase reduces all HAS but is less able to remove the larger volume completely, usually more proximally sited HAS. We should note that alteplase treatment did not independently predict reduction of multisegment HAS but there was a trend toward such an association and this analysis is underpowered. Our findings are particularly relevant given recent evidence supporting the use of endovascular clot retrieval in ischemic stroke ${ }^{18-20}$ since

Figure 2 Ordinal regression analyses for the effect of treatment on change in HAS extent from prerandomization to follow-up scan

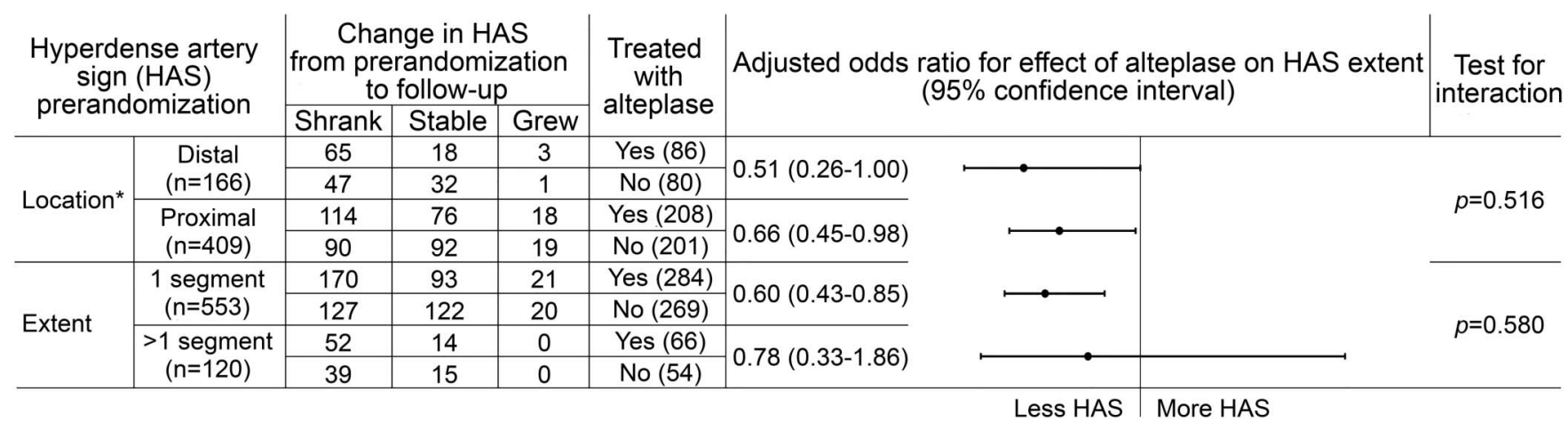

Ordinal regression analyses with change in HAS segment number (shrinkage, no change, growth) as the dependent variable assessing the importance of prerandomization HAS location (proximal = internal carotid artery, middle cerebral artery mainstem, vertebral or basilar arteries; distal = anterior or posterior cerebral arteries or sylvian branches of the middle cerebral artery) and HAS extent on the effect of alteplase. *Location analysis does not include patients with HAS in both proximal and distal arteries. Odds ratio $<1$ (left of line) indicates HAS shrinkage, $>1$ (right of line) indicates HAS growth. Results are adjusted for the effect of age, time from stroke onset to scan (hours), and NIH Stroke Scale score. 
not all patients will have the opportunity to receive, or be eligible for, such treatment. In addition, we and others $^{7,8}$ have shown improved patient outcome where HAS shrinks or disappears with IV alteplase. Conversely, increasing patient age was an independent predictor of HAS growth, which may help to explain poorer stroke outcomes in the elderly.

Our work demonstrates a novel association between IV thrombolysis and measurable growth/ shrinkage of HAS using randomized controlled trial data. We found no evidence that presence or absence of HAS materially altered the benefit of alteplase (the favorable shift in 6-month OHS). While the ordinal regression results for the HAS group alone are nonsignificant, this subgroup is underpowered and should be interpreted with caution. The appropriate interpretation is that there is no evidence of a difference in treatment effect between those with and without HAS at baseline, not that alteplase has no effect in those with HAS. To understand the apparent benefit of alteplase in patients without HAS, the presence of undetected thrombosis may be an answer; a recent report demonstrated benefit from thrombolysis for patients without apparent arterial obstruction on angiography. ${ }^{21}$

In IST-3, HAS was present in approximately $25 \%$ of patients within 6 hours of acute stroke. This is consistent with other published large series using similar scanning protocols ${ }^{22,23}$ although HAS prevalence rates of $47 \%-59 \%$ were reported in smaller studies. ${ }^{24,25}$ We confirmed that patients with HAS have more severe strokes and worse 6-month outcomes than patients without HAS. The effect of HAS presence on outcome is independent of age, time from stroke onset, stroke severity, and treatment with IV alteplase, i.e., patients with and without HAS both benefit from alteplase. Other authors have demonstrated that HAS independently predicts outcome, ${ }^{8,26,27}$ but our work also demonstrates that more extensive HAS independently predicts worse outcome among patients with the sign. We did not demonstrate an independent association between HAS location and outcome despite highly significant univariate results as demonstrated elsewhere' ${ }^{9}$ however, our multivariate analysis of outcome by HAS location is limited by the exclusion of those HAS affecting more than one arterial segment and may be underpowered.

Our study has limitations. First, some CT scans were not thin-section but prevalence rates for HAS are greater with thin-slice protocols. ${ }^{2}$ Our prerandomization HAS prevalence may therefore be an underestimate. Second, our estimates of interrater reliability ranged from $\mathrm{K}-\alpha 0.39$ to 0.46 . Prior studies have reported kappa (numerically equivalent to K- $\alpha$ ) for the identification of HAS in the 0.36-0.91 range, but these studies rarely involved more than 2 readers, whereas our panel consisted of 10 individuals masked to correlative clinical and imaging data.,28 Third, we did not define HAS based on HU measurements. Our central image analysis was performed qualitatively to reflect acute stroke care. A recent cohort study demonstrated that following IV alteplase, ischemic stroke patients with persistent arterial occlusion had lower mean density of thrombus compared to those who achieved recanalization. ${ }^{29} \mathrm{We}$ are assessing the effect of thrombus density on outcome in IST-3 in separate analyses. Fourth, we estimated hyperdensity extent based on the number of arterial segments affected. A volumetric measurement may have provided a more accurate assessment of changes in HAS volume. However, it remains unclear if HAS volume can be measured reliably. Our method of assessing thrombus extent is relevant to daily practice. Fifth, we used HAS disappearance as a surrogate indicator of arterial recanalization in the absence of angiographic data for all patients. We are analyzing available angiography data from IST-3 in a separate analysis. Sixth, multivariate models were adjusted only for variables that were associated with outcome in the main IST-3 analysis ${ }^{10}$ and for the variable time from stroke onset to scan since this latter variable would likely affect the appearance of HAS. Seventh, the limitations of the full IST-3 trial have been previously discussed ${ }^{10}$ including use of the uncertainty principle for enrolment and the potential introduction of bias through the adoption of an open design. Finally, even with 3,000 patients, IST-3 has insufficient power to reliably explore interactions between HAS and the effect of alteplase, but we found little evidence that patients with HAS responded differently to alteplase than those without HAS.

We confirmed, using data from a large randomized controlled trial, associations between the presence and extent of HAS and poor 6-month outcomes. We show clearly that alteplase accelerates shrinkage of HAS, regardless of HAS location and extent. Furthermore, we found no evidence that the improvement in functional outcome following alteplase was materially different in the presence or absence of HAS. Therefore, while HAS is useful to support a diagnosis of acute ischemic stroke and helps predict outcome, our data suggest that the presence or absence of the sign should not preclude the use of IV alteplase where clinically appropriate.

\section{AUTHOR CONTRIBUTIONS}

Grant Mair: developed study concepts and design, analyzed data, prepared the manuscript, edited and agreed on the final manuscript. Rüdiger von Kummer: developed image analysis, analyzed imaging, prepared the manuscript, edited and agreed on the final manuscript. Zoe Morris: analyzed imaging, edited and agreed on the final manuscript. Anders von 
Heijne: analyzed imaging, edited and agreed on the final manuscript. Nick Bradey: analyzed imaging, edited and agreed on the final manuscript. Lesley Cala: analyzed imaging, edited and agreed on the final manuscript. André Peeters: analyzed imaging, edited and agreed on the final manuscript. Andrew J. Farrall: analyzed imaging, edited and agreed on the final manuscript. Alessandro Adami: analyzed imaging, edited and agreed on the final manuscript. Gillian Potter: analyzed imaging, edited and agreed on the final manuscript. Geoff Cohen: analyzed data, edited and agreed on the final manuscript. Peter A.G. Sandercock: developed study concepts and design, prepared the manuscript, edited and agreed on the final manuscript. Richard I. Lindley: developed study concepts and design, edited and agreed on the final manuscript. Joanna M. Wardlaw: obtained funding, developed study concepts and design, analyzed imaging, prepared the manuscript, edited and agreed on the final manuscript, guarantor of study.

\section{ACKNOWLEDGMENT}

The IST-3 collaborative group thanks the patients who participated in the study.

\section{STUDY FUNDING}

The startup phase of IST-3 was supported by a grant from the Stroke Association, UK (TSA 04/99). The expansion phase was funded by the Health Foundation UK (2268/1282). The scan reading development was funded by Chest, Heart Stroke Scotland (R100/7). The main phase of the trial is funded by UK Medical Research Council (MRC) (grant numbers G0400069 and EME 09-800-15) and managed by NIHR on behalf of the MRC-NIHR partnership; the Research Council of Norway; Arbetsmarknadens Partners Forsakringsbolag (AFA) Insurances Sweden; the Swedish Heart Lung Fund; The Foundation of Marianne and Marcus Wallenberg, Stockholm County Council; Karolinska Institute Joint ALFproject grants Sweden; the Polish Ministry of Science and Education (grant number 2PO5B10928); the Australian Heart Foundation; Australian National Health and Medical Research Council (NHMRC); the Swiss National Research Foundation; the Swiss Heart Foundation; the Foundation for Health and Cardio-/Neurovascular Research, Basel, Switzerland; the Assessorato alla Sanita, Regione dell'Umbria, Italy; and, Danube University, Krems, Austria. Boehringer-Ingelheim GmbH donated drug and placebo for the 300 patients in the double-blind phase, but thereafter had no role in the trial. The UK Stroke Research Network (SRN study ID 2135) adopted the trial on 1/5/2006, supported the initiation of new UK sites, and in some centers, and, after that date, data collection was undertaken by staff funded by the network or working for associated NHS organizations. IST-3 acknowledges the support of the NIHR Stroke Research Network, NHS Research Scotland (NRS), through the Scottish Stroke Research Network, and the National Institute for Social Care and Health Research Clinical Research Centre (NISCHR CRC). The central imaging work was undertaken at the Brain Imaging Research Centre (www.sbirc.ed.ac.uk), a member of the Scottish Imaging Network: A Platform for Scientific Excellence (SINAPSE) collaboration (www.sinapse.ac.uk), at the Division of Clinical Neurosciences, University of Edinburgh. SINAPSE is funded by the Scottish Funding Council (SFC) and the Chief Scientist Office of the Scottish Executive (CSO). Additional support was received from Chest Heart and Stroke Scotland, DesAcc, University of Edinburgh, Danderyd Hospital R\&D Department, Karolinska Institutet, Oslo University Hospital, and the Dalhousie University Internal Medicine Research Fund.

\section{DISCLOSURE}

G. Mair reports no disclosures relevant to the manuscript. R. von Kummer has received funding from Lundbeck, Covidien, Brainsgate, and Boehringer Ingelheim. Z. Morris, A. von Heijne, N. Bradey, and L. Cala report no disclosures relevant to the manuscript. A. Peeters has received funding from Boehringer Ingelheim. A. Farrall, A. Adami, G. Potter, and G. Cohen report no disclosures relevant to the manuscript. P. Sandercock has received funding from Boehringer Ingelheim. R. Lindley has received funding from Boehringer Ingelheim and Covidien. J. Wardlaw has received funding from Medical Research
Council, Efficacy and Mechanisms Evaluation, Chest Heart Stroke Scotland. Go to Neurology.org for full disclosures.

Received April 1, 2015. Accepted in final form September 1, 2015.

\section{REFERENCES}

1. Wardlaw JM, Farrall AJ, Perry D, et al. Factors influencing the detection of early computed tomography signs of cerebral ischemia: an Internet-based, international multiobserver study. Stroke 2007;38:1250-1256.

2. Mair G, Boyd E, Chappell FM, et al. Sensitivity and specificity of the hyperdense artery sign for arterial occlusion in acute ischemic stroke. Stroke 2015;46:102-107.

3. Kharitonova T, Ahmed N, Thorén M, et al. Hyperdense middle cerebral artery sign on admission CT scan: prognostic significance for ischaemic stroke patients treated with intravenous thrombolysis in the safe implementation of thrombolysis in Stroke International Stroke Thrombolysis Register. Cerebrovasc Dis 2009;27:51-59.

4. Manelfe C, Larrue V, von Kummer R, et al. Association of hyperdense middle cerebral artery sign with clinical outcome in patients treated with tissue plasminogen activator. Stroke 1999;30:769-772.

5. Novotna J, Kadlecova P, Czlonkowska A, et al. Hyperdense cerebral artery computed tomography sign is associated with stroke severity rather than stroke subtype. J Stroke Cerebrovasc Dis 2014;23:2533-2539.

6. Nichols C, Khoury J, Brott T, Broderick J. Intravenous recombinant tissue plasminogen activator improves arterial recanalization rates and reduces infarct volumes in patients with hyperdense artery sign on baseline computed tomography. J Stroke Cerebrovasc Dis 2008;17:64-68.

7. Kharitonova T, Thoren M, Ahmed N, et al. Disappearing hyperdense middle cerebral artery sign in ischemic stroke patients treated with intravenous thrombolysis: clinical course and prognostic significance. J Neurol Neurosurg Psychiatry 2009;80:273-278.

8. Paliwal PR, Ahmad A, Shen L, et al. Persistence of hyperdense middle cerebral artery sign on follow-up CT scan after intravenous thrombolysis is associated with poor outcome. Cerebrovasc Dis 2012;33:446-452.

9. Li Q, Davis S, Mitchell P, Dowling R, Yan B. Proximal hyperdense middle cerebral artery sign predicts poor response to thrombolysis. PLoS One 2014;9:e96123.

10. The IST-3 Collaborative Group. The benefits and harms of intravenous thrombolysis with recombinant tissue plasminogen activator within $6 \mathrm{~h}$ of acute ischaemic stroke (the third international stroke trial [IST-3]): a randomised controlled trial. Lancet 2012;379:2352-2363.

11. Sandercock P, Lindley R, Wardlaw J, et al. Third International Stroke Trial (IST-3) of thrombolysis for acute ischaemic stroke. Trials 2008;9:37.

12. Wardlaw JM, Sandercock PAG, Lindley RI, et al. Association between brain imaging signs, early and late outcomes, and response to intravenous alteplase after acute ischaemic stroke in the third International Stroke Trial (IST-3): secondary analysis of a randomised controlled trial. Lancet Neurol 2015;14:485-496.

13. Wardlaw JM, von Kummer R, Farrall AJ, Chappell FM, Hill M, Perry D. A large web-based observer reliability study of early ischaemic signs on computed tomography: the Acute Cerebral CT Evaluation of Stroke Study (ACCESS). PLoS One 2010;5:e15757.

14. Wardlaw JM, von Kummer R, Carpenter T, et al. Protocol for the perfusion and angiography imaging sub-study of 
the third International Stroke Trial (IST-3) of alteplase treatment within six hours of acute ischemic stroke. Int J Stroke. Epub 2013.

15. Hayes AF, Krippendorff K. Answering the call for a standard reliability measure for coding data. Commun Methods Meas 2007;1:77-89.

16. Roozenbeek B, Lingsma HF, Perel P, et al. The added value of ordinal analysis in clinical trials: an example in traumatic brain injury. Crit Care 2011;15:R127.

17. Sandercock P, Lindley R, Wardlaw J, Whiteley W, Murray G; IST-3 Collaborative Group. Statistical analysis plan for the third International Stroke Trial (IST-3): part of a "thread" of reports of the trial. Int J Stroke 2012;7: 186-187.

18. Berkhemer OA, Fransen PS, Beumer D, et al. A randomized trial of intraarterial treatment for acute ischemic stroke. N Engl J Med 2015;372:11-20.

19. Campbell BC, Mitchell PJ, Kleinig TJ, et al. Endovascular therapy for ischemic stroke with perfusion-imaging selection. N Engl J Med 2015;372:1009-1018.

20. Goyal M, Demchuk AM, Menon BK, et al. Randomized assessment of rapid endovascular treatment of ischemic stroke. N Engl J Med 2015;372:1019-1030.

21. Lahoti S, Gokhale S, Caplan L, et al. Thrombolysis in ischemic stroke without arterial occlusion at presentation. Stroke 2014;45:2722-2727.

22. Davalos A, Toni D, Iweins F, Lesaffre E, Bastianello S, Castillo J. Neurological deterioration in acute ischemic stroke: potential predictors and associated factors in the
European cooperative acute stroke study (ECASS) I. Stroke 1999;30:2631-2636.

23. Qureshi AI, Ezzeddine MA, Nasar A, et al. Is IV tissue plasminogen activator beneficial in patients with hyperdense artery sign? Neurology 2006;66:1171-1174.

24. Kim EY, Yoo E, Choi HY, Lee JW, Heo JH. Thrombus volume comparison between patients with and without hyperattenuated artery sign on CT. AJNR Am J Neuroradiol 2008;29:359-362.

25. von Kummer R, Meyding-Lamade U, Forsting M, et al. Sensitivity and prognostic value of early CT in occlusion of the middle cerebral artery trunk. AJNR Am J Neuroradiol 1994;15:9-15.

26. Aries MJ, Uyttenboogaart M, Koopman K, et al. Hyperdense middle cerebral artery sign and outcome after intravenous thrombolysis for acute ischemic stroke. J Neurol Sci 2009;285:114-117.

27. Mustanoja S, Meretoja A, Putaala J, et al. Outcome by stroke etiology in patients receiving thrombolytic treatment: descriptive subtype analysis. Stroke 2011;42: 102-106.

28. Wardlaw JM, Mielke O. Early signs of brain infarction at CT: observer reliability and outcome after thrombolytic treatment: systematic review. Radiology 2005;235: 444-453.

29. Niesten JM, van der Schaaf IC, van der Graaf Y, et al. Predictive value of thrombus attenuation on thin-slice non-contrast CT for persistent occlusion after intravenous thrombolysis. Cerebrovasc Dis 2014;37:116-122.

\section{This Week's Neurology ${ }^{\circledR}$ Podcast}

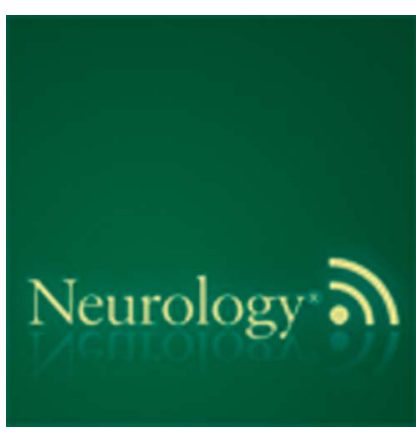

Anti-GAD antibody syndrome with concomitant cerebellar ataxia, stiff person syndrome, and limbic encephalitis (see the December 2015 issue of Neurology ${ }^{\circledR}$ Clinical Practice)

This podcast begins and closes with Dr. Robert Gross, Editor-inChief, briefly discussing highlighted articles from the January 12 , 2016, issue of Neurology. In the second segment, Dr. Alex Menze talks with Dr. Sara Schaefer about her Neurology: Clinical Practice paper on anti-GAD antibody syndrome with concomitant cerebellar ataxia, stiff person syndrome, and limbic encephalitis. Dr. Ted Burns interviews Cathy Rydell about current exciting projects happening at the AAN in our "What's Trending" feature of the week. In the next part of the podcast, Dr. Prachi Mehndiratta focuses her interview with Dr. Max Wintermark on the topic of imaging modalities used as selection criteria in endovascular stroke therapy.

Disclosures can be found at Neurology.org.

At Neurology.org, click on "RSS" in the Neurology Podcast box to listen to the most recent podcast and subscribe to the RSS feed.

No CME will be offered this week: Interviews based on articles from Neurology: Clinical Practice, Neurology ${ }^{\circledR}$ Genetics, and Neurology ${ }^{\circledR}$ Neuroimmunology \& Neuroinflammation are excluded from the CME program. 


\section{Neurology}

Effect of alteplase on the CT hyperdense artery sign and outcome after ischemic stroke Grant Mair, Rüdiger von Kummer, Zoe Morris, et al.

Neurology 2016;86;118-125 Published Online before print December 9, 2015

DOI 10.1212/WNL.0000000000002236

This information is current as of December 9, 2015

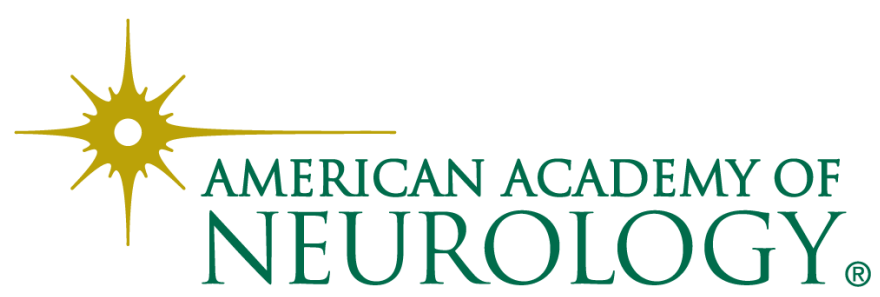




\section{Updated Information \& Services}

\section{Supplementary Material}

\section{References}

Citations

Subspecialty Collections

\section{Permissions \& Licensing}

Reprints including high resolution figures, can be found at:

http://n.neurology.org/content/86/2/118.full

Supplementary material can be found at: http://n.neurology.org/content/suppl/2015/12/09/WNL.0000000000002 236.DC1

http://n.neurology.org/content/suppl/2015/12/09/WNL.0000000000002 236.DC2

This article cites 25 articles, 10 of which you can access for free at: http://n.neurology.org/content/86/2/118.full\#ref-list-1

This article has been cited by 2 HighWire-hosted articles: http://n.neurology.org/content/86/2/118.full\#\#otherarticles

This article, along with others on similar topics, appears in the following collection(s):

\section{Class I}

http://n.neurology.org/cgi/collection/class_1

Clinical trials Randomized controlled (C̄ONSORT agreement)

http://n.neurology.org/cgi/collection/clinical_trials_randomized_control led_consort_agreement

\section{CT}

http://n.neurology.org/cgi/collection/ct

Infarction

http://n.neurology.org/cgi/collection/infarction

Information about reproducing this article in parts (figures,tables) or in its entirety can be found online at:

http://www.neurology.org/about/about_the_journal\#permissions

Information about ordering reprints can be found online:

http://n.neurology.org/subscribers/advertise

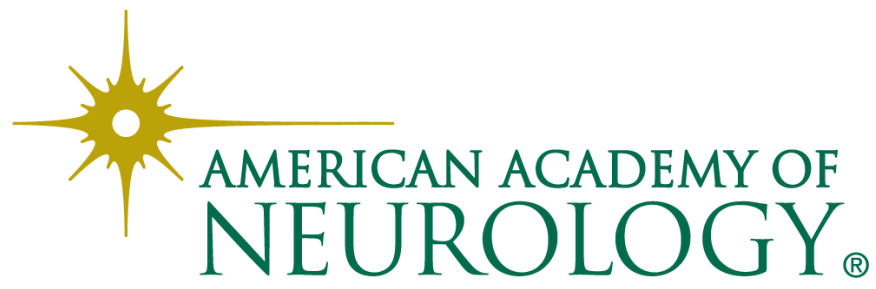

